\title{
Model predictive control system based on direct yaw moment control for 4WID self-steering agriculture vehicle
}

\author{
Hui Liu*, Shicheng Yan, Yue Shen, Chiheng Li, Yafei Zhang, Fida Hussain \\ (School of Electrical and Information Engineering, Jiangsu University, Zhenjiang 212013, Jiangsu, China)
}

\begin{abstract}
A model predictive control (MPC) approach based on direct yaw moment control (DYC) was proposed to realize the self-steering drive for a newly autonomous four-wheel independent-drive (4WID) agricultural electric vehicle. The front axle and rear axle of the vehicle chassis could rotate simultaneously around their respective center points and cut the turning radius in half at most through specific mechanical chassis structure design and four-wheel electrical drive. It had great potential to reduce wheel traffic damage to field crops if two rear electrical drive wheels can be controlled to follow wheel tracks of two front wheels during self-steering operation. Therefore, firstly, a two-degree-freedom dynamics model presenting this agricultural electric vehicle was constructed. Then, an MPC controller combined with DYC was applied to arrange torques from four wheels to match desired turning angles, direct yaw moments and travel speeds. The simulation results existed small steady error of steering angles below $0.22 \%$ as they were set at $5^{\circ}$, followed with yaw moment under $0.17 \%$ and velocity less than $1 \%$. Finally, according to experiment results, the vehicle successfully made a working turning radius of $9.1 \mathrm{~m}$ with maximum error of $0.55 \%$ when desired steering angles were $5^{\circ}$ at the speed of $1 \mathrm{~m} / \mathrm{s}$ and a minimum turning radius of $1.51 \mathrm{~m}$ with maximum error of $6.6 \%$ when steering angles were $30^{\circ}$ at the speed of $0.5 \mathrm{~m} / \mathrm{s}$. It verified that the 4 WID agricultural electric vehicle could drive autonomously and steady with small self-steering angle error under the proposed control system and has a feasibility to reduce wheel traffic damage during driving and operation.
\end{abstract}

Keywords: agriculture mechanization, 4WID electric vehicle, self-steering, model predictive control DOI: $10.25165 /$ j.ijabe.20211402.5283

Citation: Liu H, Yan S C, Shen Y, Li C H, Zhang Y F, Hussain F. Model predictive control system based on direct yaw moment control for 4WID self-steering agriculture vehicle. Int J Agric \& Biol Eng, 2021; 14(2): 175-181.

\section{Introduction}

In recent years, agricultural autonomous vehicle has become the focus of attention under the demand of improving the labor productivity and reducing the labor intensity in precision agriculture $^{[1-3]}$. Combining with the trend of agricultural automobile electrification, four-wheel independent-drive (4WID) electric vehicle could tap into potential on alleviating energy waste as well as improving the agricultural vehicle dynamics control performance $^{[4]}$.

A large number of advanced technologies have been put forward for vehicle stability control such as antilock braking system (ABS), active front steering (AFS) and direct yaw-moment control (DYC). Antilock Brake System (ABS) was able to limit yaw moment by applying brake operations on individual wheels, but could lead to energy waste ${ }^{[5]}$. Active Front Steering (AFS) was raised in $2003^{[6]}$, it could control transmission ratio between the active gear and passive rack in the steering system as the

Received date: 2019-07-11 Accepted date: 2020-07-19

Biographies: Shicheng Yan, Bachelor, research interests: agricultural robot and electrical autonomous vehicle, Email: ysc16161996@163.com; Yue Shen, PhD, Professor, research interests: agricultural robot and electrical autonomous vehicle, Email: shen@ujs.edu.cn; Chiheng Li, Bachelor, research interests: agricultural robot and active disturbance rejection control, Email: 2508476905@qq.com; Yafei Zhang, Bachelor, research interests: agricultural robot and robust adaptive control, Email: 429804349@qq.com; Fida Hussain, PhD, research interests: deep and machine learning, Email: fida.hussain07@yahoo.com.

*Corresponding author: Hui Liu, $\mathrm{PhD}$, Professor, research interests: agricultural robot, information fusion and autonomous navigation. School of Electrical and Information Engineering, Jiangsu University, Zhenjiang 212013, China. Tel: +86-13812459812, Email: amity@ujs.edu.cn. vehicle changing the speed, but rely on relatively stable working environment and vehicle center of gravity distribution. On this occasion, direct yaw moment control (DYC) was proposed with good coordination between steering angle and yaw moment ${ }^{[7-11]}$. DYC focuses on enhancing vehicle yaw stability via restraining the tendency of over-steering and under-steering to improve performance under extreme working conditions. The 4WID electric vehicle can achieve steady yaw moment and enough steering performance benefited from DYC.

Establishing a suitable model is the basis of the implementation of vehicle control system. As a variety of new structure vehicles coming up $^{[12-14]}$, their corresponding model-based control systems evolved from ordinary PID control to new algorithms ${ }^{[15,16]}$. Fuzzy control is an algorithm with strong disturbance immunity but not good at handling emergency situations, at the same time, it needs experiential knowledge to support the establishment of controller. Robust control is another algorithm that applied in motion control. It is suitable for systems with large uncertainty range and small stability margin. However, the steady-state accuracy of the robust control system is poor because it does not work in an optimal state. It is significant to choose appropriate control algorithm according to the working scenarios and accuracy requirements ${ }^{[17-19]}$. Recently, Shen et al. proposed a novel platform of 4WID electric vehicle for sprayers which showed great potential such as less press damage to crops and flexible steering performance owing to the special designed structure $^{[13]}$. Due to the double steering structure, problems like coupling between variables and difficulty in coordinated motion control are waiting to be solved. The 4WID electric vehicle was used for spraying work that owns relatively fixed motion state. 
Finally, MPC control was chosen to make optimal control scheme for pre-determined route and steering. MPC has been developed from the initial application for industrial process control to modern motion control. Due to the explicit ability to solve constraints, it has been attempted to aerospace field ${ }^{[20-22]}$. With rich theoretical experience and practical prospects on coordinated and optimized control, it has fully demonstrated great potential in complex motion control. In this case, MPC controller can optimize torque by driving the four independent motors to keep the vehicle working safely and enhance the robustness under extreme conditions ${ }^{[23-25]}$.

In the present work, a new controller was synthesized for vehicle motion control by using constrained model predictive control (MPC) and direct yaw moment Control (DYC). A novel control model was proposed based on Newtonian rigid body mechanics ${ }^{[26]}$ for this kind of 4WID self-steering vehicle to work on farm and solve steering stability problems ${ }^{[27-29]}$. Due to the special designed structure and control strategy, the proposed vehicle owned the excellent steering performance and could provide enough yaw moment for turning motion. The control problem was expressed as a Linearly Constrained Quadratic Programming (QP) to compute the optimal and dynamically-consistent front and rear axle angles required to achieve desired paths.

\section{Vehicle model and controller}

\subsection{Dynamic model of the electric-driven vehicle}

Unlike conventional electrical vehicles, this new prototype of agricultural sprayer in this paper was designed with special structural electrical-drive chassis for autonomous driving in Figure 1. The length, width and height of the sprayer were $3.20 \mathrm{~m} \times$ $1.76 \mathrm{~m} \times 2.13 \mathrm{~m}$ as shown in Figure 1a. The height of the chassis was $1.10 \mathrm{~m}$. The maximum spray range can reach $9.1 \mathrm{~m}$ when both sides of spray booms were fully unfolded. The vehicle was able to travel at a relatively low speed between $1-2 \mathrm{~m} / \mathrm{s}$ during spray operation. This agricultural electric-drive vehicle can go straight or rotate actively with four-wheel independent-drive (4WID) control strategy. The diagram of the electric-drive vehicle chassis was shown in Figure 1b. Each of the four wheels was independently driven by a set of hub motor. The front axle was conjunctive with the front left wheel and the front right wheel, and so as the rear axle set shown in Figure 1b. The front axle and rear axle were linked the vehicle chassis, and both axles can rotate around their respective center points flexibly. $W$ stands for the lateral distance $(\mathrm{m})$ between two front/rear wheels in Figure 1b. $L$ represents the longitudinal distance $(\mathrm{m})$ from the center of the front axle to that of the rear axle. $l_{f}$ and $l_{r}$ stands for longitudinal distances $(\mathrm{m})$ from the front and rear axle to the center of gravity (CG) of vehicle, respectively. $T_{1}, T_{2}, T_{3}$, and $T_{4}$ represented hub-motor driven torques $(\mathrm{N} \cdot \mathrm{m})$ of the front left, front right, rear left and rear right wheels, respectively. $\alpha$ and $\beta$ are the yaw angles $\left({ }^{\circ}\right)$ of front axle and rear axle, respectively. $R$ represented for the self-steering turning radius.

Due to the flexible rotation design of the front axles and rear axles, the agricultural electrical vehicle has the potential ability to reduce traffic damage to crops from wheels rolling and improve spray effect if rear-wheel tracks could follow front-wheel tracks precisely when making turns or changing lanes. That is, the 4-wheel tracks could be decreased to 2-wheel tracks to protect crops from overmuch rolling. Thus, the yaw angles of the front axle $\alpha$ and the rear axle $\beta$ of vehicle should be same so that the tracks are coincident in Figure 1b. Therefore, in this paper, a two-degree-freedom dynamics model of this electrical-drive vehicle was presented for self-steering controller design, as shown in Figure 2. The model parameter variables of this electrical-drive vehicle were shown in Table 1.

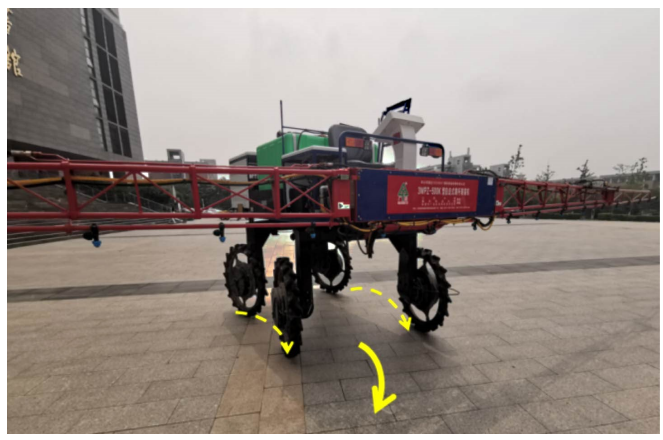

a. New prototype of sprayer with electric-drive chassis

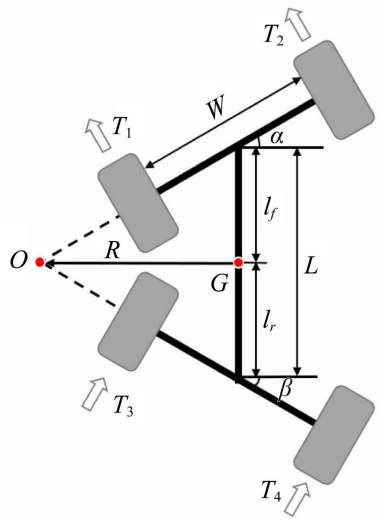

b. Diagram of self-steering 4 in-wheel motors independent-drive chassis

Figure 1 Electric-drive 4WID sprayer and its chassis self-steering structure diagram

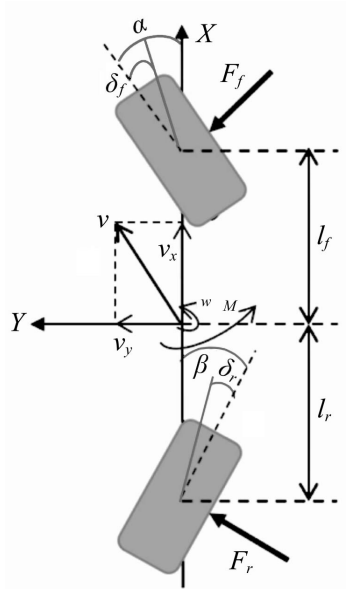

Figure 2 Two-degree-freedom monorail model of the vehicle

Traditional control systems usually take force models into consideration which rely on the force between earth and wheels, but it is limited by measuring time-varying and complex variable influenced by environment factors like friction and slip rate. In this case, the agricultural vehicle was designed to run at low speed and more attention was paid for self-steering axle angles that would determine the turning process. The angle of each axle could be controlled by motor torques that depended on electric current. The four wheels of vehicle driven by four independent hub motors can provide suitable torques to front and rear axles by closed loop controlling of input voltages. 
Table 1 Parameters of the vehicle model

\begin{tabular}{|c|c|c|}
\hline Variables & Physical meaning & Unit \\
\hline$M$ & Yaw moment of vehicle & $\mathrm{N} \cdot \mathrm{m}$ \\
\hline$v$ & Velocity at center of gravity (CG) of vehicle & $\mathrm{m} \cdot \mathrm{s}^{-1}$ \\
\hline$v_{x}$ & Longitudinal velocity at CG of vehicle & $\mathrm{m} \cdot \mathrm{s}^{-1}$ \\
\hline$v_{y}$ & Transverse velocity at CG of vehicle & $\mathrm{m} \cdot \mathrm{s}^{-1}$ \\
\hline$w$ & Self-steering angular velocity & $\mathrm{rad} \cdot \mathrm{s}^{-1}$ \\
\hline$V_{1}, V_{2}, V_{3}, V_{4}$ & $\begin{array}{l}\text { Velocity of left front wheel } \\
\text { Velocity of right front wheel } \\
\text { Velocity of left rear wheel } \\
\text { Velocity of right rear wheel }\end{array}$ & $\mathrm{m} \cdot \mathrm{s}^{-1}$ \\
\hline$l_{f}, l_{r}$ & $\begin{array}{l}\text { Longitudinal distance from the front axle to } C G \\
\text { Longitudinal distance from the rear axle to } C G\end{array}$ & $\mathrm{~m}$ \\
\hline$\delta_{f}, \delta_{r}$ & $\begin{array}{l}\text { Front steering angle } \\
\text { Rear steering angle }\end{array}$ & $\operatorname{deg}$ \\
\hline$W, L$ & $\begin{array}{l}\text { Lateral distance between two front /rear wheels } \\
\text { Longitudinal distance from the center of the front } \\
\text { axle to the center of the rear axle }\end{array}$ & $\mathrm{m}$ \\
\hline$\alpha, \beta$ & $\begin{array}{l}\text { Yaw angle of front axle of vehicle } \\
\text { Yaw angle of rear axle of vehicle }\end{array}$ & $\operatorname{deg}$ \\
\hline
\end{tabular}

$J_{\alpha}$ and $J_{\beta}$ are the rotational inertia $\left(\mathrm{kg} \cdot \mathrm{m}^{2}\right)$ of front axle and rear axle of the vehicle, respectively. $\mu_{\alpha}$ and $\mu_{\beta}$ are the coefficient of friction of the front and rear axles, respectively. The torque equations can be concluded as follow:

$$
\begin{aligned}
& J_{\alpha} \ddot{\alpha}+\mu_{\alpha} \dot{\alpha}=T_{2}-T_{1} \\
& J_{\beta} \ddot{\beta}+\mu_{\beta} \dot{\beta}=T_{3}-T_{4}
\end{aligned}
$$

In practice, the brushless hub DC motors are controlled by PWM and can be regarded as DC motor.

$$
\begin{gathered}
T_{j}=K_{T} u_{j}-K_{T} K_{\varphi} n_{j} \\
n_{j}=K_{\varphi}^{-1}\left(u_{j}-I_{j a} R_{j a}\right)
\end{gathered}
$$

where, $K_{\varphi}=C_{e} \phi$ is electrical constant of each motor, N.m/A; $C_{e} \phi$ are motor parameters that can be measured; $\phi$ is magnetic flux; $C_{e}$ is a constant; $K_{T}=9.55 K_{\varphi} / R_{a} ; u_{j}, n_{j}$ and $T_{j}$ are the voltage $(\mathrm{V})$, motor speed $(\mathrm{r} / \mathrm{min})$, and torque of the motors $(\mathrm{N} \cdot \mathrm{m})$, respectively. $I_{j a}$ and $R_{j a}$ are the indication of the armature current and internal resistance of four motors, respectively.

According to the control strategy of direct yaw moment control (DYC), the compensating yaw moment of vehicle generated by in-wheel motors can be derived

$$
M=l_{f} K_{w} \delta_{f}-l_{r} K_{w} \delta_{r}
$$

where, $K_{w}$ is the cornering stiffness of front and rear wheels, $\mathrm{N} / \mathrm{rad}$; Assume that steering angle is small and vehicle velocity $v$ is slow, the front and rear steering angles $\delta_{f}, \delta_{r}$ can be shown as below:

$$
\left\{\begin{array}{l}
\delta_{f}=\alpha-\left(v_{y}+l_{f} w\right) / v_{x} \\
\delta_{r}=-\beta-\left(v_{y}+l_{r} w\right) / v_{x}
\end{array}\right.
$$

Consider $l_{f}=l_{r}=\frac{L}{2}$, the yaw moment equation can be written as

$$
M=\frac{L K_{w}}{2}(\alpha+\beta)
$$

The angular velocity $w$ and the turning radius $R$ of the electrical vehicle can be written as

$$
\begin{gathered}
w=\frac{v_{x} \delta_{f}}{L\left(1+K_{f} v_{x}^{2}\right)}+\frac{v_{x} \delta_{r}}{L\left(1+K_{r} v_{x}^{2}\right)} \\
R=\frac{v_{x}}{w}=\frac{L\left(1+K_{f} v_{x}^{2}\right)}{\delta_{f}}+\frac{L\left(1+K_{r} v_{x}^{2}\right)}{\delta_{r}}
\end{gathered}
$$

where, $K_{f}$ and $K_{r}$ are the stability factors, their equations can be written as

$$
\left\{\begin{array}{l}
K_{f}=\frac{m}{W^{2} K_{w}}(\alpha-\beta) \\
K_{r}=\frac{m}{W^{2} K_{w}}(\beta-\alpha)
\end{array}\right.
$$

\subsection{Linearization and optimization of equations}

The differential equations were established and simplified from the model. Linear optimization of axle rotation differential equations can be expressed as follow:

$$
\begin{aligned}
& \ddot{\alpha}=-\frac{\mu_{\alpha}}{J_{\alpha}} \dot{\alpha}-\frac{K_{T}}{J_{\alpha}} u_{1}+\frac{K_{T}}{J_{\alpha}} u_{2} \\
& \ddot{\beta}=-\frac{\mu_{\beta}}{J_{\beta}} \dot{\beta}-\frac{K_{T}}{J_{\beta}} u_{3}-\frac{K_{T}}{J_{\beta}} u_{4}
\end{aligned}
$$

Consider angular velocity $w$ and turning radius $R$ of the vehicle under the control rate $(\alpha \approx \beta)$, linearization and simplification of $w$, $R$ are available as follow:

$$
\begin{gathered}
w=\frac{\left(\delta_{f}+\delta_{r}\right)}{L} v_{x} \\
R=\frac{L}{\delta_{f}+\delta_{r}}
\end{gathered}
$$

It can be inferred that this vehicle would perform well in turning test since the angular velocity $w$ is doubled, and the turning radius $R$ is halved compared with traditional vehicles $\left(\delta_{r}=0\right.$, $\left.w=\frac{v_{x} \delta_{f}}{L}, R=\frac{L}{\delta_{f}}\right)^{[29]}$.

Consider $r$ is the radius of each wheel, the vehicle would move slowly and the transverse velocity $v_{y}$ would tend to 0 under working condition. The velocity of the vehicle can be considered as $v_{x}$,

$$
v_{x}=\frac{\pi r \cos \alpha}{2 K_{\varphi}}\left(u_{1}+u_{2}\right)+\frac{\pi r \cos \beta}{2 K_{\varphi}}\left(u_{3}+u_{4}\right)
$$

The nonlinear model of $v_{x}$ above is expressed as the following form,

$$
v_{x}=f(x, u), x \in R^{n_{x}}, u \in R^{n_{u}}
$$

where, $n_{x}$ and $n_{u}$ represent the dimensions of $x$ and $u$, linearizing it with Taylor formula,

$$
v_{x}=f\left(x_{k}, u_{k}\right)+A_{v}\left(x-x_{k}\right)+B_{v}\left(u-u_{k}\right)+e_{k}
$$

where, $x_{k}$ and $u_{k}$ are control state vector and inputs at the sample time $k$, which can be measured; $A_{v}=\left.\frac{\partial f}{\partial x}\right|_{x_{k}, u_{k}}$ and $B_{v}=\left.\frac{\partial f}{\partial u}\right|_{x_{k}, u_{k}}$ are partial of $f(x, u)$ with respect to $x$ and $u ; e_{k}$ is a higher order infinitesimal of variable.

$$
\begin{aligned}
& A_{v}=\left[-\sin \alpha_{k} \frac{\pi r}{2 K_{\varphi}}\left(u_{1 k}+u_{2 k}\right)-\sin \beta_{k} \frac{\pi r}{2 K_{\varphi}}\left(u_{3 k}+u_{4 k}\right) \quad 0 \quad 0\right] \\
& B_{v}=\left[\begin{array}{llll}
\frac{\pi r}{2 K_{\varphi}} \cos \alpha_{k} & \frac{\pi r}{2 K_{\varphi}} \cos \alpha_{k} & \frac{\pi r}{2 K_{\varphi}} \cos \beta_{k} & \frac{\pi r}{2 K_{\varphi}} \cos \beta_{k}
\end{array}\right] \\
& f\left(x_{k}, u_{k}\right)=\cos \alpha_{k} \frac{\pi r}{2 K_{\varphi}}\left(u_{1 k}+u_{2 k}\right)+\cos \beta_{k} \frac{\pi r}{2 K_{\varphi}}\left(u_{3 k}+u_{4 k}\right) \\
& d_{v}=\sin \alpha_{k} \frac{\pi r}{2 K_{\varphi}}\left(u_{1 k}+u_{2 k}\right) \alpha_{k}+\sin \beta_{k} \frac{\pi r}{2 K_{\varphi}}\left(u_{1 k}+u_{2 k}\right) \beta_{k}
\end{aligned}
$$

where, $u_{n k}$ represents the output of motor $n$ at the working time $k$; $\alpha_{k}$ and $\beta_{k}$ represent desired yaw angle values of $\alpha$ and $\beta$ at the working time $k ; v_{x}$ can be reduced as: 


$$
v_{x}=\left[\begin{array}{llll}
\frac{\pi r}{2 K_{\varphi}} & \frac{\pi r}{2 K_{\varphi}} & \frac{\pi r}{2 K_{\varphi}} & \frac{\pi r}{2 K_{\varphi}}
\end{array}\right] u
$$

\subsection{MPC controller design}

In this research work, MPC was proposed to control four independent motor with the state space equations.

$$
\left\{\begin{array}{l}
\dot{x}=A x+B u \\
y=C x+D u
\end{array}\right.
$$

where, $x$ is the state vector of the vehicle; $\dot{x}$ is the rate of change in the state variables; $A$ is the corresponding state vector matrix; $B$ is the matrix of control variables; $y$ is the observation of output variables and $C$ is the output feedback matrix. Consider the state vector $x=[\alpha, \beta, \dot{\alpha}, \dot{\beta}]^{\mathrm{T}}$, system input $u=\left[u_{1}, u_{2}, u_{3}, u_{4}\right]^{\mathrm{T}}$, system output $y=\left[\alpha, \beta, M, v_{x}\right]^{\mathrm{T}}$.

$$
\begin{gathered}
A=\left[\begin{array}{cccc}
0 & 0 & 1 & 0 \\
0 & 0 & 0 & 1 \\
0 & 0 & -\frac{\mu_{\alpha}}{J_{\alpha}} & 0 \\
0 & 0 & 0 & -\frac{\mu_{\beta}}{J_{\beta}}
\end{array}\right], B=\left[\begin{array}{cccc}
0 & 0 & 0 & 0 \\
0 & 0 & 0 & 0 \\
-\frac{K_{T}}{J_{\alpha}} & \frac{K_{T}}{J_{\alpha}} & 0 & 0 \\
0 & 0 & \frac{K_{T}}{J_{\beta}} & -\frac{K_{T}}{J_{\beta}}
\end{array}\right], \\
C=\left[\begin{array}{cccc}
1 & 0 & 0 & 0 \\
0 & 1 & 0 & 0 \\
\frac{L}{2} K_{w} & \frac{L}{2} K_{w} & 0 & 0 \\
0 & 0 & 0 & 0
\end{array}\right], D=\left[\begin{array}{cccc}
0 & 0 & 0 & 0 \\
0 & 0 & 0 & 0 \\
0 & 0 & 0 & 0 \\
\frac{\pi r}{2 K_{\varphi}} & \frac{\pi r}{2 K_{\varphi}} & \frac{\pi r}{2 K_{\varphi}} & \frac{\pi r}{2 K_{\varphi}}
\end{array}\right]
\end{gathered}
$$

Consider that the control horizon of MPC is $m$ and the prediction horizon is $p$, where $(m<p) . \quad(k+1 \mid k)$ stands the prediction $(k+1)$ based on current data $(k)$. The system can be described by quadratic form indicators to make outputs approach to target.

$$
\min J=\| \Gamma_{y}\left[\left(Y_{p}(k+1 \mid k)-R(k+1)\right]\left\|^{2}+\right\| \Gamma_{u} \Delta U(k) \|^{2}\right.
$$

Subject to

$$
\begin{aligned}
& \Delta u_{\min } \leq \Delta u(k+j \mid k) \leq \Delta u_{\max }, j=0,1, \ldots, m-1 \\
& y_{\min } \leq y(k+j \mid k) \leq y_{\max }, j=0,1, \ldots, p
\end{aligned}
$$

where, $R(k)=[\alpha, \beta, M, v]^{\mathrm{T}}$ is a given reference; $\Gamma_{y}=[3,3,5,3]^{\mathrm{T}}$ and $\Gamma_{u}=[1,1,1,1]^{\mathrm{T}}$ are the weighting factors of $\mathrm{y}$ and $\Delta U(k) ; u_{\min }$ is the minimum voltage $(-72 \mathrm{~V})$ of the motor; $u_{\max }$ is the maximum voltage $(+72 \mathrm{~V})$ of the motor; $\Delta u_{\min }, \Delta u_{\max }$ are the minimum $(-10 \mathrm{~V})$ and maximum $(+10 \mathrm{~V})$ value of variations of the system input voltages, $y_{\min }=[-30,-30,0,-10]^{\mathrm{T}}$ and $y_{\max }=[+30,+30,7000$, $+10]^{\mathrm{T}}$ are the minimum and maximum value of the system output variables, respectively.

Subtracting the target $R(k)$ from the output $Y_{p}(k)$, smaller difference value leads to better optimization accuracy. To achieve this, MPC was used to solve finite-horizon optimal control problem at each step.

\section{Simulation and experiment results}

In order to evaluate the performance of the proposed self-steering MPC control system, the 4WID agricultural vehicle model with MPC controller was built with Matlab Simulink. The main parameters of this 4WID vehicle model and the MPC controller used in the simulation are shown in Tables 2 and 3. In the simulation test of fastness and accuracy for MPC-based self-steering system, the reference values of the front-axle yaw angle $\alpha$ and rear-axle yaw angle $\beta$ were $\alpha, \beta=5^{\circ}$, yaw moment of vehicle $M=6000 \mathrm{~N} \cdot \mathrm{m}$, and longitudinal vehicle velocity $v_{x}=2 \mathrm{~m} / \mathrm{s}$ (Table 3). The sample time for MPC controller was $0.2 \mathrm{~s}$. The prediction horizon was 15 and the control horizon was 3 . The weighting factors of front-axle yaw angle $\alpha$, rear-axle yaw angle $\beta$,

\begin{tabular}{|c|c|c|}
\hline Variables & Description of model parameters & Values \\
\hline$J_{\alpha}$ & Rotational inertia of front axle $/ \mathrm{kg} \cdot \mathrm{m}^{2}$ & 800 \\
\hline$J_{\beta}$ & Rotational inertia of rear axle $/ \mathrm{kg} \cdot \mathrm{m}^{2}$ & 800 \\
\hline$l_{f}$ & Longitudinal distance from the front axle to $\mathrm{CG} / \mathrm{m}$ & 0.79 \\
\hline$l_{r}$ & Longitudinal distance from the rear axle to $\mathrm{CG} / \mathrm{m}$ & 0.79 \\
\hline$K_{w}$ & Cornering stiffness of the front and rear wheels $/ \mathrm{N} \cdot \mathrm{rad}^{-1}$ & 3000 \\
\hline$W$ & Lateral distance between two front and rear wheels $/ \mathrm{m}$ & 1.51 \\
\hline$L$ & $\begin{array}{l}\text { Longitudinal distance from the center of the front axle to } \\
\text { the center of the rear axle/m }\end{array}$ & 1.58 \\
\hline$K_{\varphi}$ & Electrical constant of each motor $/ \mathrm{N} \cdot \mathrm{m} \cdot \mathrm{A}^{-1}$ & 0.88 \\
\hline$K_{T}$ & Electromagnetic torque constant $/ \mathrm{N} \cdot \mathrm{m} \cdot \mathrm{V}^{-1}$ & 12.5 \\
\hline
\end{tabular}
longitudinal vehicle velocity $v_{x}$ and yaw moment $M$ for MPC system outputs were 3, 3, 5 and 3, respectively. An impact of disturbance was simulated to test the MPC system robustness. It happened at $10 \mathrm{~s}$ with the amplitude of $2^{\circ}$ on axle $\alpha$, which was $40 \%$ of initial reference.

Table 2 Main parameter values for the 4WID vehicle model

Table 3 MPC controller parameters for 4WID vehicle self-steering

\begin{tabular}{clc}
\hline Variables & \multicolumn{1}{c}{ Description of control parameters } & Values \\
\hline$T_{s}$ & Sample time/s & 0.2 \\
$p$ & Prediction horizon & 15 \\
$m$ & Control horizon & 3
\end{tabular}

Initial motor voltage of front-left wheel,

$U_{0}=\left[u_{1}, u_{2}, u_{3}, u_{4}\right]^{\mathrm{T}} \quad$ front-right wheel, rear-left wheel, rear-right $\quad 0,0,0,0$ wheel/V

System reference outputs of front-axle yaw $5\left(^{\circ}\right), 5\left(^{\circ}\right)$

$R=[\alpha, \beta, M, v]^{\mathrm{T}} \quad$ angle $\alpha$, rear-axle yaw angle $\beta$, yaw moment $6000(\mathrm{~N} \cdot \mathrm{m})$, $M$, longitudinal vehicle velocity $v_{x} \quad 2\left(\mathrm{~m} \cdot \mathrm{s}^{-1}\right)$

output weighting factors of front-axle yaw angle $\alpha$, rear-axle yaw angle $\beta$, yaw moment $\quad 3,3,5,3$ $M$, longitudinal vehicle velocity $v_{x}$

$\Gamma_{u} \quad$ output weighting factors of system inputs $u_{1}, \quad 1,1,1,1$

Disturbance time and amplitude of disturbing yaw angle $10(\mathrm{~s}), 2\left(^{\circ}\right)$

In this dynamic simulation model, assuming that sideslip does not occur under the direction of effective DYC, the friction is considered to be the only force of power that supports motion action. It means this system was designed to keep motion pose in low velocity condition and the control variables would be at small value during the steady state.

From the simulation results, the inputs and outputs of the 4WID vehicle are shown in Figure 3. It can be seen that the input voltages of four hub motors showed duality for two left wheels $\left(u_{1}\right.$, $u_{3}$ ) and two right wheels $\left(u_{2}, u_{4}\right)$, while the maximum and minimum of $u_{1}, u_{3}$ are shown at point $\mathrm{A}$ and $\mathrm{C}$, and the maximum and minimum of $u_{2}, u_{4}$ are shown at point $\mathrm{B}$ and $\mathrm{D}$ in Figure 3a. The front-axle yaw angle $\alpha$, the rear-axle yaw angle $\beta$, the yaw moment $M$ and vehicle velocity $v$ are shown in Figure $3 \mathrm{~b}$.

In static state test $(0-10 \mathrm{~s})$, the front-axle yaw angle $\alpha$, the rear-axle yaw angle $\beta$ and the yaw moment $M$ reached to peak value within $6 \mathrm{~s}$ with $3 \%$ overshoot, and the rise time of those were shown at point $\mathrm{A}, \mathrm{C}$ and $\mathrm{E}$ in Figure $3 \mathrm{~b}$, respectively. At the moment of $6 \mathrm{~s}$, the vehicle velocity was still in the acceleration phase, shown at point $\mathrm{G}$ in Figure 3b. All state variables reached steady state at $7.56 \mathrm{~s}$, which was equivalent to point $\mathrm{F}$ in Figure $3 \mathrm{~b}$. It cost $7.56 \mathrm{~s}$ to achieve the desired yaw angles and velocity when the vehicle started to run under the proposed MPC system with 
target condition.

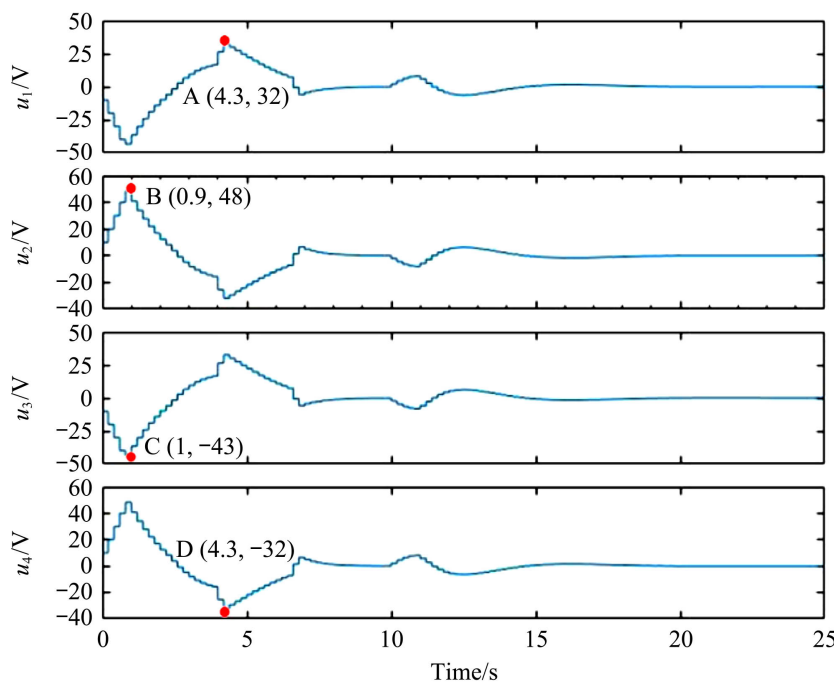

a. Input voltages of four hub motors $\left(u_{1}\right.$ : front-left wheel, $u_{2}$ : front-right wheel, $u_{3}$ : rear-left wheel, $u_{4}$ : rear-right wheel)
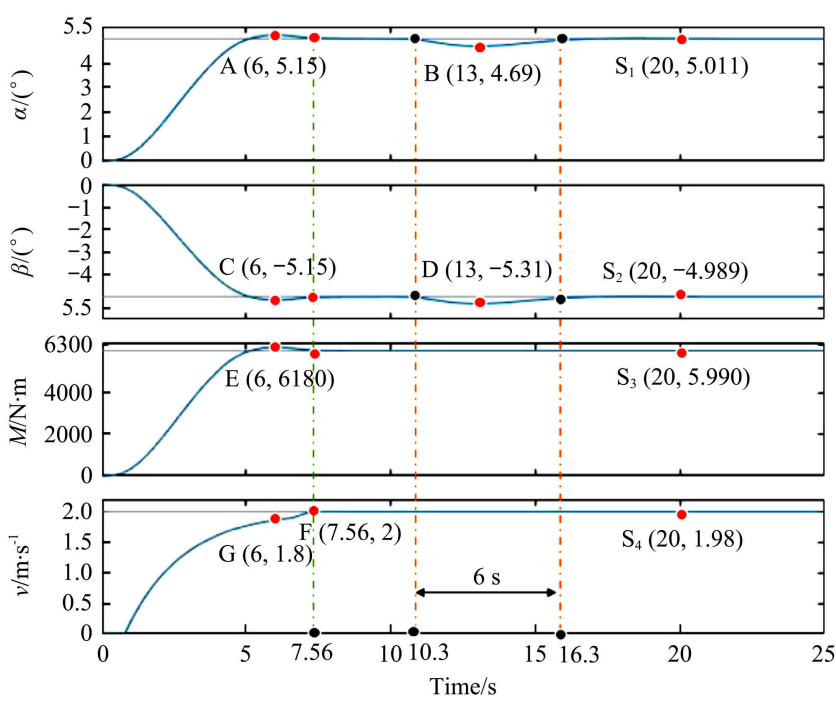

b. MPC system outputs ( $\alpha$ : front-axle yaw angle, $\beta$ : rear-axle yaw angle, $M$ : yaw moment, $v$ : longitudinal vehicle velocity)

Figure 3 Simulation results of MPC controller for turning path at $v_{x}=2 \mathrm{~m} / \mathrm{s}$

In dynamic state test $(10-20 \mathrm{~s})$, a pulse disturbance of front-axis yaw angle $\alpha$ with the amplitude of $2^{\circ}$ at the time of $10 \mathrm{~s}$ was generated in the simulation system to test the robustness of the proposed MPC controller. It can be seen in Figure $3 b$ that the MPC system reacted quickly. The front-axis yaw angle $\alpha$ and the rear-axle yaw angle $\beta$ started to fluctuate at $10.3 \mathrm{~s}$ under the effect of disturbance, but they returned to desired value by $10.6 \mathrm{~s}$ promptly owing to the proposed the MPC controller. For better visual comparison between front and rear yaw angle, the front-axle yaw angle $\alpha$ was shown as its actual value, while the rear-axle yaw angle $\beta$ was shown as the negative of its actual value, in Figure $3 b$. The peak values of $\alpha$ and $\beta$ during this process were shown at point $\mathrm{B}$ and D. Both front-axle and rear-axle yaw angle errors were under $6.2 \%$ when this pulse disturbance magnitude was $40 \%$ of reference value. The longitudinal vehicle velocity $v$ and yaw moment $M$ remained unchanged during disturbance response. So far, the proposed MPC system showed good robustness on yaw moment and yaw angle control, which owed to the advantages of DYC set in MPC.

In steady state test (over $20 \mathrm{~s}$ ), the system outputs $\alpha, \beta, M$ and $v$ reached stable status after $20 \mathrm{~s}$, which are shown at point S1-S4. The steady state errors of $\alpha, \beta, M$ and $v$ were $0.22 \%, 0.22 \%, 0.17 \%$ and $1.00 \%$, respectively. In general, the front-axle yaw angle $\alpha$, the rear-axle yaw angle $\beta$, the yaw moment $M$ and vehicle velocity $V$ reacted promptly to reach stable status and compensated disturbance with the proposed MPC system.

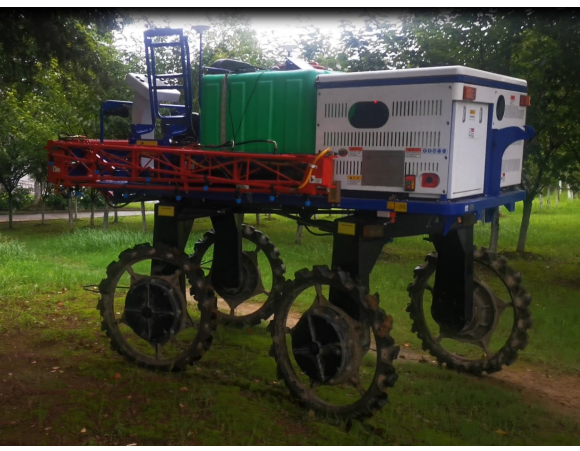

a. Electric sprayer with 4WID chassis

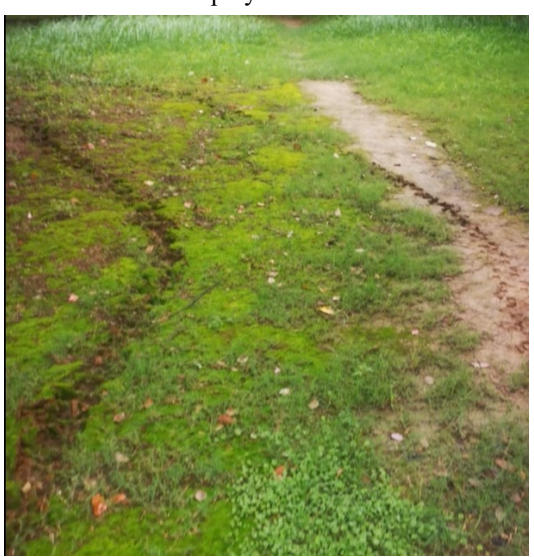

b. Experiment site

Figure 4 Self-steering agriculture vehicle experiment of MPC controller

Based on the simulation results, a self-steering evaluation experiment was conducted to verify the effectiveness of the proposed two-degree-freedom dynamics model and the MPC control system of this electrical-drive vehicle. The 4WID agricultural electric vehicle and the experiment environment were shown in Figure 4. The test platform of the agricultural electric sprayer was equipped with GPS navigation system, and the navigation data was transferred to PC with a RS232 serial port. The state parameters of the vehicle model can be managed through an on-board PC and were sent to the real-time MPC controller.

For the experiment part, two groups of self-steering angles were set: (1) The front-axle yaw angle $\alpha$ and rear-axle yaw angle $\beta$ were $\alpha, \beta=5^{\circ}$, and such the desired turning radius $R$ was $9.1 \mathrm{~m}$ based on Equation (14); (2) $\alpha, \beta=30^{\circ}$, and $R=1.51 \mathrm{~m}$; They were represented for common yaw angles and large yaw angles of this electric vehicle with the specific chassis structure, respectively. The low vehicle velocity $\left(v_{x}=1 \mathrm{~m} / \mathrm{s}\right.$ and $\left.v_{x}=0.5 \mathrm{~m} / \mathrm{s}\right)$ was adopted for field experiments due to security reason and the physical limitation of test site. In this way, following the proposed 4WID vehicle model and the MPC controller, the electric sprayer was expected to move and turn around as desired steering angle and turning radius.

The experimental results about the self-steering condition under the MPC controller were illustrated as follows. As can be seen from Figures $5 \mathrm{a}$ and $5 \mathrm{c}$, the actual front and rear yaw angles of the autonomous vehicle varied with time and tended to the target steering angles of $5^{\circ}$ and $30^{\circ}$, respectively. Correspondently, the turning radiuses of self-driving approached to the desired $9.1 \mathrm{~m}$ and 
$1.51 \mathrm{~m}$, respectively, as seen in Figures $5 \mathrm{~b}$ and $5 \mathrm{~d}$. The front-axle yaw angle was shown as its actual value, and the rear-axle yaw angle was shown as the negative of its actual value in Figure 5, for better visual comparison between front and rear yaw angles, as in the simulation result analysis in Figure $3 \mathrm{~b}$. In Figure 5a, the electric vehicle traveled at the speed of $1 \mathrm{~m} / \mathrm{s}$ and turned in a circle with relatively stable steering angles close to $5^{\circ}$ after $4 \mathrm{~s}$ under the MPC controller. The front-axis yaw angle existed $4.2 \%$ of overshoot and $0.6 \%$ of steady state error, while the rear-axis yaw angle showed $7.0 \%$ overshoot and $0.6 \%$ steady state error for $\alpha, \beta=5^{\circ}$. The actual turning radius was between $9.05 \mathrm{~m}$ and $9.15 \mathrm{~m}$

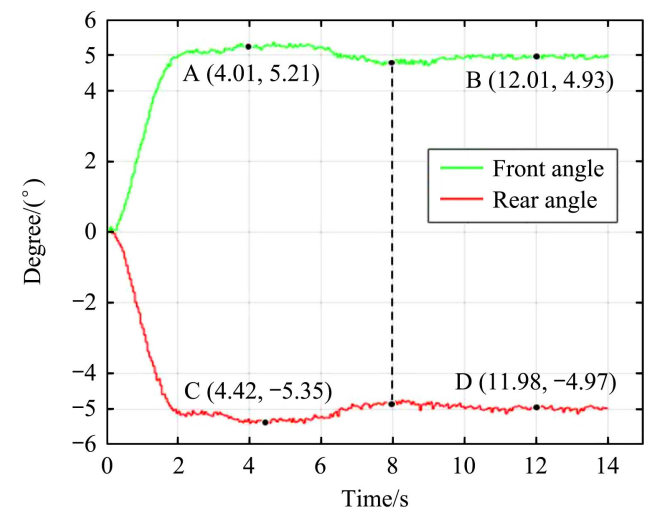

a. Actual front-axis and rear-axis yaw angles for $\alpha, \beta=5^{\circ}$

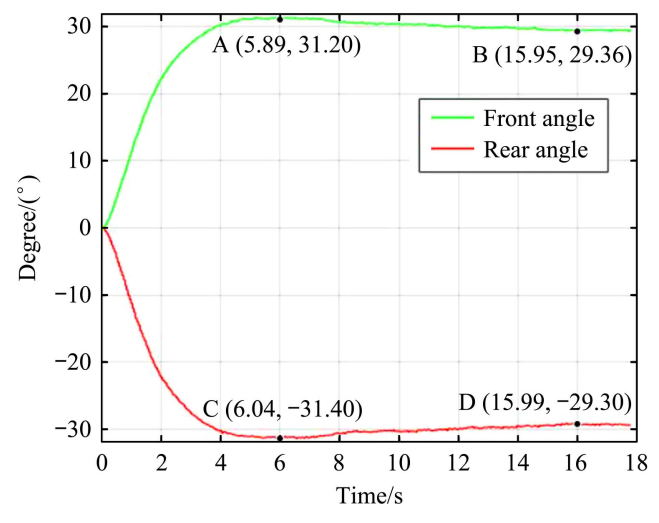

c. Actual front-axis and rear-axis yaw angles for $\alpha, \beta=30^{\circ}$ with the maximum error of $0.55 \%$ when the desired value was $9.1 \mathrm{~m}$, as seen in Figure 5b. As for $\alpha, \beta=30^{\circ}$, in Figure $5 \mathrm{c}$, the relatively large yaw angles that the electric vehicle were expected to achieve, the front-axle yaw angle existed $0.7 \%$ of overshoot and $2.13 \%$ of steady state error, while the rear-axle yaw angle showed $1.3 \%$ of overshoot and $2.3 \%$ of steady state error. Under this condition, as the vehicle traveled at $0.5 \mathrm{~m} / \mathrm{s}$, the turning radius increased to $1.6 \mathrm{~m}$ and then reduced to $1.5 \mathrm{~m}$ with the maximum error of $6.6 \%$ comparing with the desired turning radius of $1.5 \mathrm{~m}$, which matched the variation of the steering angle changes, as seen in Figure 5d.

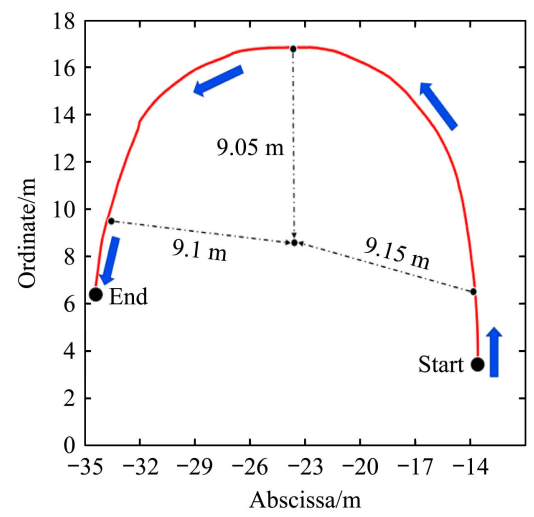

b. Actual turning path for $R=9.1 \mathrm{~m}$

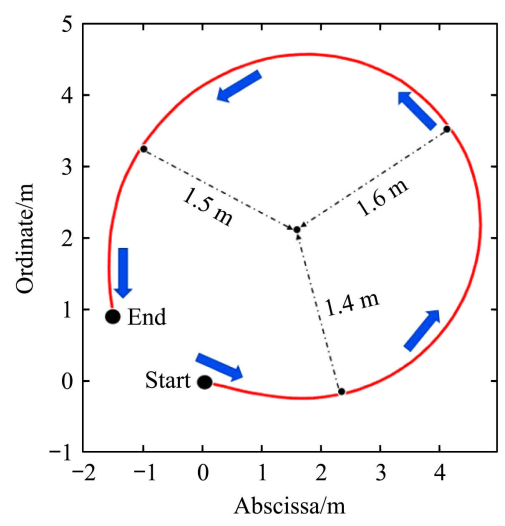

d. Actual turning path for $R=1.51 \mathrm{~m}$

Figure 5 Experimental results for self-steering agriculture vehicle under the proposed MPC controller

\section{Conclusions}

In this research, a control system combined with MPC and DYC was proposed for self-steering of agricultural 4WID electric sprayer. The mathematical model was built based on the rigid body mechanics. To avoid being affected by unmeasured environment factors as the ordinary solutions did, the original model relying on frictional force was replaced with torque-based model derived from four independent wheels. Thus, the pose of front and rear axles can be controlled by the current of four motors.

The proposed control system was built to manipulate the turning motion of the agricultural electric vehicle, and the final target was to perform spray operation on the farm. By following a preset route, the vehicle can spray the whole farmland by driving straight lines and making turns with circular motions. It means that the main focus of control was to complete the specified circular motion with small positioning error. For this vehicle, the state values were confirmed when the vehicle trajectory was set by the on-board computer. So, the controller was considered as successfully built as the trajectory error was very low in the experiment. According to the experimental results, the vehicle axles showed good coordination and following performance. The tests of two turning paths were made successfully with $0.55 \%$ maximum error for turning radius of $9.1 \mathrm{~m}$ under normal motion condition and $6.6 \%$ maximum error for turning radius $1.51 \mathrm{~m}$ under the minimum turning radius mode. Therefore, the agricultural electrical vehicle with the proposed control system can reduce traffic damage to crops from wheels rolling because the rear-wheel tracks could follow front-wheel tracks precisely when making turns.

Combined with the actual vehicle working condition, this spray vehicle will be equipped with a large water tank, which could lead to gravity center changing during spraying operation. Thus, the MPC controller may face slight model mismatch. Equation (18) is a nonlinear expression simplified by Taylor formula at the operating point with steering angles of $5^{\circ}$. When the turning angles of movement were away from the working point, for example at the steering angles of $30^{\circ}$, the system may face slight model mismatch. It also explained the existence of relatively larger trajectory deviation in minimum turning radius experiment. 
Future work: This system can be improved by doing some supplement in the future, such as adding adaptive control algorithm in extreme environment. In another way, adding visual feedback from upper computer with real-time correction could further reduce the tracking error. In addition, it is significant to set $\mathrm{GC}$ at the center of the vehicle, or the rotational inertia of axles will move if the front and rear axles exist large weight difference.

\section{Acknowledgements}

This research work was funded by the National Natural Science Foundation of China (Grant No. 51975260), Jiangsu Provincial Key Research and Development Program (Grant No. BE2018372), Jiangsu Natural Science Foundation (Grant No. BK20181443), Zhenjiang City Key Research and Development Program (Grant No. NY2018001), Qing Lan Project of Jiangsu Province, the Priority Academic Program Development (PAPD) of the Jiangsu Higher Education Institutions, China. The authors would like to acknowledge the other colleagues of the team for providing assistance in the experiment.

\section{[References]}

[1] Liu Z, Zheng W, Wang N, Lyu Z, Zhang W. Trajectory tracking control of agricultural vehicles based on disturbance test. Int J Agric \& Biol Eng, 2020; 13(2): 138-145.

[2] Ryabov V V, Kniaziuk T V, Mikhailov M S, Motovilina G D, Khlusova E I. Structure and properties of new wear-resistant steels for agricultural machine building. Inorganic Materials Applied Research, 2017; 8(6): 827-836.

[3] Zhou Q Q, Xue X Y, Qin W C, Cai C, Zhou L F. Optimization and test for structural parameters of UAV spraying rotary cup atomizer. Int $\mathrm{J}$ Agric \& Biol Eng, 2017; 10(3): 78-86.

[4] Pei W, Lan Y B, Luo X W, Zhou Z Y, Wang Y. Integrated sensor system for monitoring rice growth conditions based on unmanned ground vehicle system. Int J Agric \& Biol Eng, 2014; 7(2): 75-81.

[5] Singh K B, Arat M A, Taheri S. An intelligent tire based tire-road friction estimation technique and adaptive wheel slip controller for antilock brake system. Journal of Dynamic Systems, Measurement, and Control, 2013; 135(3): 31002-31002.

[6] Doumiati M, Sename O, Dugard L, Martinez-Molina J J, Gaspar P, Szabo Z. Integrated vehicle dynamics control via coordination of active front steering and rear braking. European Journal of Control, 2013; 19(2): $121-143$.

[7] Zhao C, Guo L. PID controller design for second order nonlinear uncertain systems. Science China Information Sciences, 2017; 60: 022201.

[8] Wang Y, Jin Q, Zhang R. Improved fuzzy PID controller design using predictive functional control structure. ISA Transactions, 2017; 71(2): 354-363.

[9] Mahto T, Mukherjee V. Fractional order fuzzy PID controller for wind energy based hybrid power system using quasi-oppositional harmony search algorithm. IET Generation Transmission \& Distribution, 2017; 11(13): 3299-3309.

[10] Fernandes H R, Garcia A P. Design and control of an active suspension system for unmanned agricultural vehicles for field operations. Biosystems Engineering, 2018; 174: 107-114.

[11] Julián S H, Rincón V J, Francisco P, Francisco A, Fernando C. Field evaluation of a self-propelled sprayer and effects of the application rate on spray deposition and losses to the ground in greenhouse tomato crops. Pest Management Science, 2011; 67(8): 942-947.

[12] Qiu Q, Fan Z, Meng Z, Zhang Q, Cong Y, Li B, et al. Extended Ackerman Steering Principle for the coordinated movement control of a four wheel drive agricultural mobile robot. Computers and Electronics in Agriculture, 2018; 152: 40-50.

[13] Shen Y, Zhang B, Liu H, Cui Y, Hussain F, He S, et al. Design and development of a novel independent wheel torque control of $4 \mathrm{WD}$ electric vehicle. Mechanics, 2019; 25(3): 210-218.

[14] Cao D, Tang B, Jiang H, Yin C, Zhang D, Huang Y. Study on low-speed steering resistance torque of vehicles considering friction between tire and pavement. Applied Science, 2019; 9: 1015

[15] Yadbantung R, Bumroongsri P. Tube-based robust output feedback MPC for constrained LTV systems with applications in chemical processes. European Journal of Control, 2019; 47: 11-19.

[16] Alamir M. Numerical investigation regarding an MPC scheme with non uniformly weighted stage cost without terminal constraints: Application to the control of a real-life cryogenic plant. IFAC Papers Online, 2018; 51(20): 418-423.

[17] Magdy G, Shabib G, Elbaset A A, Mitani Y. Frequency stabilization of renewable power systems based on MPC with application to the Egyptian Grid. IFAC Papers Online, 2018; 51(28): 280-285.

[18] Villanueva M E, Quirynen R, Diehl M, Chachuat B, Houska B. Robust MPC via min-max differential inequalities. Automatica, 2017; 77(C): 311-321.

[19] Li D, Xia Y, Gao Y, Cannon M. Distributed stochastic mpc of linear systems with additive uncertainty and coupled probabilistic constraints. IEEE Transactions on Automatic Control, 2017; 62(7): 3474-3481.

[20] Bujarbaruah M, Zhang X, Borrelli F. Adaptive MPC with chance constraints for FIR systems. 2018 Annual American Control Conference (ACC), Milwaukee, WI, 2018; pp.2312-2317.

[21] Wen H, Cong M, Wang G, Qin W, Xu W, Zhang Z. Dynamics and optimized torque distribution based force position hybrid control of a 4-DOF redundantly actuated parallel robot with two point-contact constraints. International Journal of Control, Automation and Systems, 2019; 17(5): 1293-1303.

[22] Li P, Yang Z, Jiang S. Needle-tissue interactive mechanism and steering control in image-guided robot-assisted minimally invasive surgery: a review. Medical \& Biological Engineering \& Computing, 2018; 56(6): 931-949.

[23] Dehghani H, Welch C R, Pourghodrat A, Nelson C A, Oleynikov D, Dasgupta $\mathrm{P}$, et al. Design and preliminary evaluation of a self-steering, pneumatically driven colonoscopy robot. Journal of Medical Engineering \& Technology, 2017; 41(3): 223-236.

[24] Zhao Z, Du R. Yaw moment control strategy for four wheel side driven EV. Automatic Control \& Computer Sciences, 2018; 52(1): 32-39.

[25] Ding S, Lu L, Wei X Z. Sliding mode direct yaw-moment control design for in-wheel electric vehicles. IEEE Transactions on Industrial Electronics, 2017; 64(8): 6752-6762.

[26] Kobayashi T, Katsuyama E, Sugiura H, Ono E, Yamamoto M. Direct yaw moment control and power consumption of in-wheel motor vehicle in steady-state turning. Vehicle System Dynamics, 2017; 55(1): 104-120.

[27] Guo J, Luo Y, Li K, Dai Y. Coordinated path-following and direct yaw-moment control of autonomous electric vehicles with sideslip angle estimation. Mechanical Systems \& Signal Processing, 2018; 105: 183-199.

[28] Jing Z, Wong P K, Ma X, Xie Z. Chassis integrated control for active suspension, active front steering and direct yaw moment systems using hierarchical strategy. Vehicle System Dynamics, 2017; 55(1): 72-103.

[29] Yu S Y, Wang J, Wang Y, Chen H. Disturbance observer based control for four wheel steering vehicles with model reference. IEEE/CAA Journal of Automatica Sinica, 2018; 5(6): 1121-1127. 